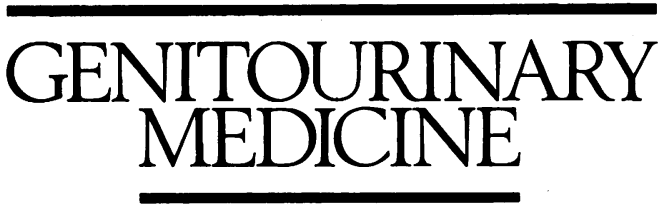

Early warning

From 1998 the title of

Genitourinary Medicine

will become

\title{
Sexually Transmitted Infections
}

\section{Surveillance of antibiotic susceptibility of Neisseria gonorrhoeae in the Western Pacific}

Surveillance is a "good thing," at least if you're an epidemiologist. But what is it for? In particular, what is an international laboratory based surveillance programme in sexually transmitted diseases for?

A recent review in these pages suggested that surveillance programmes could be used to determine the need for public health action, to assess the effectiveness of programmes, to set priorities for planning and resource allocation, and to define high risk subgroups for intervention. ${ }^{1}$ These are all laudable objectives. The trouble is, one can't do all of them well at the same time, particularly if one doesn't have much money. So what are the actual purposes of the international laboratory surveillance of sexually transmitted diseases?

On page 355, Tapsall and colleagues ${ }^{2}$ report the 1992-4 results from the WHO Western Pacific Region Gonococcal Antimicrobial Surveillance Programme (GASP) on antibiotic susceptibility in Neisseria gonorrhoeae. The authors and the WHO are to be congratulated on bringing off a collaborative surveillance project in a group of countries separated by enormous distances and having wide variation in economic development. Regional surveillance is clearly not easy.

In fact surveillance at the regional level might be thought of as a "North-South" problem, in that countries with large budgets and high technological capacities are collaborating with countries having not many public health resources. (Actually in this Australian led case it's a "South-North" problem.) Benefits must flow in both directions to make such collaborations possible. Typically, technical assistance and laboratory supplies flow in the direction of the less developed countries, and information flows back towards the richer countries. This is not always an easy transaction, and there aren't a lot of international resources available for it. Thus, international surveillance has often tended to be improvised and patchy. Sometimes a surveillance programme can lack focus until a problem is perceived to be acute, at which point it may well be too late.
International surveillance for gonorrhoea can't really be justified by the belief that gonorrhoea control will cut the incidence of new infections of HIV, as the authors suggest. Although at least one well known study in Africa suggests that STD control can decrease HIV infection, ${ }^{3}$ and although we would all devoutly wish this generally to be the case, such interventions are not going to be made at the regional level and won't depend on regional registries. Surveillance for resistance in gonorrhoea needs a justification of its own.

Probably this kind of surveillance should be thought of as having two different raisons d'etre, from the two points of view involved. From the point of view of the less developed countries, there is the chronic problem of improving national STD control programmes. From the point of view of the richer countries, there is the acute problem (at least in historical perspective) of monitoring the worldwide spread of antibiotic resistance. It is notable that the GASP project reports a broad increase in quinolone resistant gonoccoci in the Western Pacific Region during 1992-4. Twelve of 13 reporting centres saw at least low level quinolone resistance in 1994. Seven centres noted high level resistance and the rate of high level resistance rose significantly in the region over the reporting period.

Relatively high rates of resistance to quinolones have been reported previously from Japan, ${ }^{4}$ the Philippines ${ }^{5}$ and Hong Kong, ${ }^{6}$ all in the Western Pacific Region, as well as in Thailand $^{7}$ and Rwanda. ${ }^{8}$ But it is sobering to see the increase in a region-wide format. Both penicillinase producing $N$ gonorrhoeae (PPNG) and chromosomally mediated penicillin and tetracyclin resistant $N$ gonorrhoeae (CMRNG) were seen in Pacific countries before their arrival in Australia, the United Kingdom, and the United States. In the United States, PPNG was reported in the mid 1970 s and CMRNG in the mid 1980s. As a recent report noted, these events "heralded the end of an era in which gonorrhoea could be confidently treated with relatively inexpensive antibiotics such as penicillin and tetracyclines".9 
Thus, in 1989, broad spectrum cephalosporins and quinolones were recommended as the primary therapies for gonorrhoea in the United States. ${ }^{10}$ In the early 1990 s, gonoccoccal strains with reduced susceptibility to the quinolones were reported in Australia, Europe, and the United States as occasional imports, usually from Asian countries. By 1994 the prevalence of less sensitive strains in Ohio was large enough to suspect endemicity. ${ }^{11}$ Recently, the US Centers for Disease Control Gonococcal Isolate Surveillance Project (GISP) reported an increase in the US prevalence of isolates with decreased quinolone sensitivity from $0.4 \%$ in 1991 to $1.3 \%$ in 1994 , with occasional fully resistant isolates. This finding, while not sufficient to cause a change in CDC recommendations, sends a warning ripple across the surface of gonorrhoea treatment in the United States. The authors of the GISP report recommend that in areas like Cleveland (where $17.5 \%$ of reported isolates had decreased susceptibility to ciprofloxacin in 1994) gonorrhoea should be treated with a third generation cephalosporin or with spectinomycin.

Fortunately, reports that quinolone resistance might be associated with ceftriaxone resistance via a multiple drug resistance mechanism such as a membrane pump ${ }^{12}$ do not appear to have been confirmed. ${ }^{9}$ However, the recent first appearance of the long predicted vancomycin resistant strain of MRSA in Japan ${ }^{13}$ suggests that in the case of antibiotic resistance, a version of the ontological argument for the existence of God applies: if you can imagine it, it probably exists. Tapsall et al are right that cephalosporin sensitivities of gonococci should be closely monitored in the Western Pacific.

The agenda of the developed countries, then (and these clearly include the United States in the present instance), is close monitoring of a rather important series of events in the rapid evolution of antibiotic resistance. This probably fits best under determining the need for public health action in the list of uses of surveillance programmes given above, since ideally the developed countries would be interested in intervention in the less developed countries, on the grounds that it's better to stop it there than stop it here. For a surveillance project like GASP, such a goal would imply increased attention to the completeness of regional reporting and to the comparability of sampling plans and reporting locations between time periods. Viewed from the other perspective, improvement of treatment in the less developed countries in the region would imply increased representativeness of the data at national levels as well as the continuation of GASP's technical assistance efforts. The surveillance goal here would be to assess the effectiveness of programmes. The WHO should keep supporting GASP and should think carefully about its double function in the next few years.

Department of Epidemiology and Biostatistics,

University of Califormia, San Francisco, and

Medical Service, San Francisco General Hospital,

San Francisco, California, USA

1 Catchpole MA. The role of epidemiology and surveillance systems in the control of sexually transmitted diseases. Genitourin Med 1996;72:321-9. WHO Western Pacific Region Gonoccocal Antimicrobial Surveillance

Wre Programme. Survellance of antibiotic susceptibility of Neisseria gonorrhoeae in the WHO Western Pacific Region 1992-4. Genitourin Med 1997;73:355-61.

3 Grosskurth H, Mosha F, Todd J, Mwijarubi E, Klokke A, Senkoro K, et al. Impact of improved treatment of sexually transmitted diseases on HIV infection in rural Tanzania: randomised controlled trial. Lancet 1995; 346:530-6.

4 Tanaka M, Kumazawa J, Matsumoto T, Kobyashi I. High prevalence of Neisseria gonorrhoeae strains with reduced susceptibility to fluoroquinolones in Japan. Genitourin Med 1994;70:90-3.

5 Knapp JS, Mesola VP, Neal SW, Wi TE, Tuazon C, Manalastas R, et al. Molecular epidemiology, in 1994, of Neisseria gonorrheae in Manila and Cebu City, Republic of the Philippines. Sex Transm Dis 1997;24:2-7.

$6 \mathrm{Kam} \mathrm{KM}$, Lo KK, Ng KYH, Cheung MM. Rapid decline in pencillinaseproducing Neisseria gonorrhoeae in Hong Kong associated with emerging 4-fluoroquinolone resistance. Genitourin Med 1995;71:141-4.

7 Knapp JS, Wongba C, Limpakarnjanarat K, Young NL, Parekh MC, Neal $\mathrm{SW}$, et al. Antimicrobial susceptibilities of strains of Neisseria gonorrheae in Bangkok, Thailand: 1994-1995. Sex Transm Dis 1997;24:142-8.

8 Bogaerts J, Tello WM, Akingeneye J, Mukantabana V, Van Dyck E, Piot P. Effectiveness of norfloxacin and ofloxacin for treatment of gonorrhoea affectiveness of norfloxacin and ofloxacin for treatment of gonorrhoea and increase of in vitro susceptibility

9 Fox KK, Knapp JS, Holmes KK, Hook EW 3d, Judson FN, Thompson $\mathrm{SE}$, et al. Antimicrobial resistance in Neisseria gonorrheae in the United States, 1988-1994: the emergence of decreased susceptibility to the fluoroquinolones. F Infect Dis 1997;175:1396-403.

10 Centres for Disease Control and Prevention. 1989 sexually transmitted disease treatment guidelines. MMWR 1989;38:1-43.

11 Ohye R, Higa H, Vogt R, Doyle L, Sayers D, Halpin DJ. Decreased susceptibility of Neisseria gonorrheae to fluoroquinolones-Ohio and Hawaii, 1992-1994. ЭAMA 1994;271:1733-4.

12 Deguchi T, Saito I, Tanaka M, Sato K, Deguchi K, Yasuda M, et al. Fluoroquinolone treatment failure in gonorrhea. Emergence of a Neisseria gonorrheae strain with enhanced resistance to fluoroquinolones. Sex Transm Dis 1997;24:247-50.

13 Antibiotic resistance hogs the limelight. (News. Science and Medicine) Lancet 1997;349:1674. 